\title{
Chromosomal instability in untreated primary prostate cancer as an indicator of metastatic potential
}

Eric T. Miller ${ }^{1 \dagger}$, Sungyong You ${ }^{2 \dagger}$, Radu M. Cadaneanu' ${ }^{1}$, Minhyung Kim³ ${ }^{3}$ Junhee Yoon ${ }^{3}$, Sandy T. Liu ${ }^{1,4}$, Xinmin Li ${ }^{5,6}$, Lorna Kwan ${ }^{1}$, Jennelle Hodge ${ }^{2}$, Michael J. Quist ${ }^{4}$, Catherine S. Grasso ${ }^{3}$, Michael S. Lewis ${ }^{7}$, Beatrice S. Knudsen ${ }^{2}$, Michael R. Freeman ${ }^{2}$ and Isla P. Garraway ${ }^{1,6,8^{*}}$

\begin{abstract}
Background: Metastatic prostate cancer (PC) is highly lethal. The ability to identify primary tumors capable of dissemination is an unmet need in the quest to understand lethal biology and improve patient outcomes. Previous studies have linked chromosomal instability (CIN), which generates aneuploidy following chromosomal missegregation during mitosis, to PC progression. Evidence of CIN includes broad copy number alterations (CNAs) spanning > 300 base pairs of DNA, which may also be measured via RNA expression signatures associated with CNA frequency. Signatures of CIN in metastatic PC, however, have not been interrogated or well defined. We examined a published 70-gene CIN signature (CIN70) in untreated and castration-resistant prostate cancer (CRPC) cohorts from The Cancer Genome Atlas (TCGA) and previously published reports. We also performed transcriptome and CNA analysis in a unique cohort of untreated primary tumors collected from diagnostic prostate needle biopsies (PNBX) of localized (M0) and metastatic (M1) cases to determine if CIN was linked to clinical stage and outcome.
\end{abstract}

Methods: PNBX were collected from 99 patients treated in the VA Greater Los Angeles (GLA-VA) Healthcare System between 2000 and 2016. Total RNA was extracted from high-grade cancer areas in PNBX cores, followed by RNA sequencing and/or copy number analysis using OncoScan. Multivariate logistic regression analyses permitted calculation of odds ratios for CIN status (high versus low) in an expanded GLA-VA PNBX cohort $(n=121)$.

(Continued on next page)

\footnotetext{
* Correspondence: igarraway@mednet.ucla.edu

${ }^{*}$ Eric T. Miller and Sungyong You contributed equally to this work.

'Department of Urology, David Geffen School of Medicine at UCLA, Box

951738, 10833 Le Conte Ave 66-188 CHS UCLA, Los Angeles, CA 90095, USA

${ }^{6}$ Jonsson Comprehensive Cancer Center, David Geffen School of Medicine at UCLA, Box 951738, 10833 Le Conte Ave 66-188 CHS UCLA, Los Angeles, CA 90095, USA
}

Full list of author information is available at the end of the article

(c) The Author(s). 2020 Open Access This article is licensed under a Creative Commons Attribution 4.0 International License, which permits use, sharing, adaptation, distribution and reproduction in any medium or format, as long as you give appropriate credit to the original author(s) and the source, provide a link to the Creative Commons licence, and indicate if changes were made. The images or other third party material in this article are included in the article's Creative Commons licence, unless indicated otherwise in a credit line to the material. If material is not included in the article's Creative Commons licence and your intended use is not permitted by statutory regulation or exceeds the permitted use, you will need to obtain permission directly from the copyright holder. To view a copy of this licence, visit http://creativecommons.org/licenses/by/4.0/ The Creative Commons Public Domain Dedication waiver (http://creativecommons.org/publicdomain/zero/1.0/) applies to the data made available in this article, unless otherwise stated in a credit line to the data. 


\begin{abstract}
(Continued from previous page)
Results: The CIN70 signature was significantly enriched in primary tumors and CRPC metastases from M1 PC cases. An intersection of gene signatures comprised of differentially expressed genes (DEGs) generated through comparison of M1 versus MO PNBX and primary CRPC tumors versus metastases revealed a 157-gene "metastasis" signature that was further distilled to 7-genes (PC-CIN) regulating centrosomes, chromosomal segregation, and mitotic spindle assembly. High PC-CIN scores correlated with CRPC, PC-death and all-cause mortality in the expanded GLA-VA PNBX cohort. Interestingly, approximately 1/3 of M1 PNBX cases exhibited low CIN, illuminating differential pathways of lethal PC progression.
\end{abstract}

Conclusions: Measuring CIN in PNBX by transcriptome profiling is feasible, and the PC-CIN signature may identify patients with a high risk of lethal progression at the time of diagnosis.

Keywords: Prostate cancer, Metastases, Chromosomal instability, CIN, Prostate needle biopsies, TCGA

\section{Background}

Chromosomal instability (CIN) describes a cell state where whole chromosomes or chromosome arms are gained, lost, or develop structural aberrations at high rates $[1,2]$. Chromosome missegregation due to mitotic errors is the root cause of CIN and contributes to the overall genomic instability that propels tumor evolution [3-7]. The two main products of CIN are aneuploidy, reflected in whole chromosome and arm-level (broad) copy number alterations (CNAs), and micronuclei, which are extra-nuclear bodies containing chromosomal segments that are prone to rupture, eliciting further DNA damage and inflammatory responses [5, 7-11]. Aneuploidy is a well-recognized hallmark of cancer and is detectable in $88 \%$ of samples in The Cancer Genome Atlas (TCGA) [11]. Aneuploidy frequency correlates with TP53 mutations, overall mutation rate (after excluding tumors with high microsatellite instability), and proliferative gene expression signatures [11-13]. Interestingly, there is an inverse correlation between aneuploidy levels and leukocyte infiltration, which may have implications related to tumor immunogenicity [11, 14]. Activation of the cyclic GMP-AMP synthase-stimulator of interferon genes (cGAS-STING) pathway by cytoplasmic DNA spillage from ruptured micronuclei can drive metastatic spread through downstream non-canonical NF-KB signaling in cell line models that display high chromosomal missegregation [15].

Despite implications that CIN may be a catalyst for genomic alterations and a permissive environment for tumor progression, quantitation of CIN in tumors is rarely performed due to technical challenges and lack of therapeutic implications $[10,16,17]$. To facilitate measurement of CIN, which can be costly using exome sequencing or single nucleotide polymorphism (SNP) arrays, computational approaches can be used to derive gene expression signatures as a surrogate of genomic CIN measurements. Accordingly, a validated 70-gene CIN expression signature of (CIN70) has been shown to be consistently associated with poor outcome across a variety of tumors $[15,16]$. CIN70 was generated by calculating total chromosomal imbalance via spectral karyotype and SNP-Chip analysis, followed by identification of corresponding differentially expressed genes (DEGs) and predicted poor outcome in twelve independent data sets representing six cancer types. Annotation of CIN70 DEGs revealed many with roles in chromosomal replication/condensation/separation, mitotic spindle assembly, and centrosome function [16].

In prostate cancer (PC), metastatic and castration resistant tumors exhibiting features of genomic instability as a consequence of DNA damage repair (DDR) defects has led to promising clinical trials evaluating inhibitors that target these genomic subgroups [18-21]. In contrast, the prevalence, molecular mechanisms, and impact of CIN as a prognostic indicator and/or therapeutic target in PC have lagged, despite detection of aneuploidy in a large proportion of $\mathrm{PC}$, including untreated primary tumors and mCRPC [11, 22, 23]. Recently, a transcriptome profiling method capable of estimating the number of altered chromosome arms in PC samples from TCGA was described [23]. Application of this method to surgical specimens (radical prostatectomy and transurethral resection of the prostate) from two independent PC cohorts with long-term follow-up available suggested that broad CNAs were associated with an increased risk of PC lethality. Taken together, these observations implicate CIN is a potential catalyst of $\mathrm{PC}$ progression through genomic and structural chromosomal aberrations and warrants further exploration for clinical utility.

Here, we aimed to evaluate the prevalence of CIN across the clinical spectrum of $\mathrm{PC}$, including localized castration-sensitive PC (CSPC), metastatic CSPC (mCSPC) and mCRPC. A large volume of genomic and transcriptomic data from PC patients was utilized to assess CIN, including a rare collection of diagnostic PNBX $(n=99)$ comprised of patients with de novo metastatic (clinical stage M1) as well as presumed localized (clinical stage M0) high-grade CSPC assembled from patients treated over a period of 15 years at a 
single Veterans hospital. Although $<6 \%$ of new PC diagnoses are stage M1, a disproportional number of PC-related deaths occur in these men who exhibit a 5year survival rate of approximately $28 \%$ [24]. The high lethality of M1 cases suggests a potential role for CIN in the development/propagation of aggressive subtypes. Consequently, profiling cases of de novo metastatic PC may reveal CIN as a useful biomarker of dissemination, progression, and/or inherent treatment resistance that is feasible to measure in diagnostic PNBX and may reveal biology leading to new therapeutic targets.

\section{Methods}

\section{Ethics statement}

We received institutional review board (IRB) approval to abstract data and procure both fresh and archival PNBX samples from men diagnosed and treated within the Greater Los Angeles VA (GLA-VA) Healthcare System (protocol numbers PCC2018-020201 and PCC201011489). Informed consent was obtained for all prospectively collected tissue specimens. A consent waiver was approved for collection of archival samples and data. All specimens were stripped of personal health information and identifiers.

\section{GLA-VA patient cohort}

After Institutional Review Board approval was obtained, 99 PC cases with archival biopsy tissue available were selected for RNA sequencing (RNAseq) from a total cohort 1927 cases identified within the GLA-VA cancer registry or procedure logs. The selected cases were divided into sub-cohorts based upon tumor burden at diagnosis or recurrence/progression documented in imaging reports, including ${ }^{99 \mathrm{~m}} \mathrm{Tc}$-methylene disphosphonate $\left({ }^{99 \mathrm{~m}} \mathrm{Tc}-\mathrm{MDP}\right)$ planar bone scintigraphy, ${ }^{18} \mathrm{~F}-\mathrm{NaF}$ positron emission tomography $\left({ }^{18} \mathrm{~F}-\mathrm{NaF} \mathrm{PET}\right)$, ultrasounds, magnetic resonance imaging (MRI), computed tomography $(\mathrm{CT})$ scans, and plain radiographs. Cases were designated clinical stage M1 if metastatic lesions were identified on imaging scans performed within 1 year of the diagnostic PNBX. Osteoblastic, osteolytic, and/or sclerotic bone lesions observed on bone scan were confirmed or by the presence of overlapping lesions on plain radiographs, CT or MRI imaging. Oligometastatic "oligo" disease was defined by $\leq 5$ extrapelvic lymph node and/or bone metastases and no visceral metastases. Polymetastatic "poly" disease was defined as $>5$ metastases or any visceral involvement. M1 cases were designated M1-oligo or M0-poly based on the aforementioned tumor burden assessment. Cases that were considered M0 at diagnosis, but demonstrated eventual metastatic progression (M0-M) were designated M0oligo or M0-poly based on tumor burden on imaging scans at the time of follow-up. Cases were designated as clinical stage $\mathrm{M} 0$ non-metastatic (M0-NM) if no metastatic lesions were identified at the time of last follow-up. If imaging scans were not performed, or equivocal results were obtained, the cases were categorized as MX and excluded from analysis. A significant subset of M0 cases from the original 1927 cohort did not have an indication for diagnostic imaging due to diagnosis of low- or intermediate-risk PC according to D'Amico classification [25].

\section{Sample processing and RNA analysis}

Diagnostic hematoxylin and eosin (H\&E) PNBX slides were reviewed by a genitourinary pathologist, and highgrade tumor areas (Gleason grade 4, 5, or neuroendocrine/small cell) were encircled. Transfer of annotated areas to the paraffin-embedded tissue blocks was performed and 1-2 mm sterile circular biopsy punches enabled manual procurement of formalin-fixed tissue from the block. A minimum of $2(1 \mathrm{~mm})$ cancer cores were used from each high-grade tumor area for RNA and/or DNA preparation. Total RNA was extracted from tissue cores using the Ambion Recover All Total Nucleic Acid Isolation Kit for FFPE (ThermoFisher). Samples were eluted with $\mathrm{H}_{2} \mathrm{O}$ and quantitated with nanodrop and bioanalyzer. Approximately 40-100 ng of RNA was used to generate the libraries using the TruSeq RNA Access Library Prep Kit (Illumina) according to manufacturer's instructions. Quality control for libraries was performed with the HS DNA Qubit and bioanalyzer, followed by sequencing on the Hiseq3000 at $1 \times 50$.

\section{OncoScan CNA analysis}

DNA was extracted from $1 \mathrm{~mm}$ FFPE cores with AnaPrep 12 Automated Nucleic Acid Preparation Instrument (Biochain) and quantitated with Qubit (Life technology). Approximately $80 \mathrm{ng}$ of gDNA was used in the Affymetrix OncoScan FFPE Assay kit (Affymetrix) according to manufacturer's instructions. The Affymetrix GeneChip Scanner 3000 G7 was used and data was processed with Affymetrix Chromosome Analysis Suite 3.1 (ChAS 3.1) to generate probe level and gene level $\log 2$-ratio using normalized data.

\section{Sequencing data access}

Gene expression data and OncoScan CNA data were deposited into the GEO database with accession GSE147493 and GSE147353, respectively.

\section{Genomic alterations of the TCGA PC cohort}

We computed scores for focal CNAs, broad CNAs, overall CNAs (broad and focal), TMB, genomic fusion, MSI, PC-CIN, and CIN70 using data from 473 prostate samples in TCGA. We used GISTIC 2.0 [26] to compute focal, broad and overall CNAs. CNAs with the GISTIC value less than -0.3 were categorized as 'loss' and larger 
than 0.3 as 'gain'. If the length of altered (loss or gain) regions was longer than $98 \%$ of the chromosomal arm, it was classified as broad CNAs, while less than 98\% chromosomal arm were classified as focal CNAs. For each case, the broad CNA score was generated by calculating the total number of broad CNAs over the total number of chromosomal arms, and the focal CNA score was calculated based upon the number of focal CNAs over the total number of genes. MSI score was based upon the MANTIS scores as described in previously published reports [27]. We obtained masked copy number segment, Mutect2 result of somatic mutation from UCSC Xena Browser (http://xena.ucsc.edu/). Fusion data was obtained from a published report [28]. TMB score was calculated from counts of non-synonymous mutations in a sample divided by exon size per million bases (total exon size $=299.029409 \mathrm{MB}$ ). Fusion score was defined as the ratio of the number of fusion events and the total number of fusion types [28]. The signature activation scores were computed using a Z-score method [29].

\section{RNAseq data analysis of PNBX cohort}

For RNAseq data analysis, the quality of sequence reads from the RNAseq data were assessed and low quality reads were filtered using the FastQC tool (Babraham Bioinformatics, Cambridge, UK) and ShortRead (v. 1.30.0) package from $\mathrm{R}$ bioconductor (version 3.3). Quantification of gene level expression from preprocessed RNA-seq results were performed with the UCSC hg19 build of the Homo sapiens genome, through the use of the Subread aligner and the featureCounts software [30, 31]. To reduce systemic bias between samples, the Trimmed Mean Method (TMM) was applied to gene level expression counts [32]. Genes were filtered out and excluded from downstream analysis if they failed to achieve raw read counts of at least 2 across all the libraries. Differentially expressed genes (DEGs) were determined with false discovery rate $(\mathrm{FDR})<0.05$ and fold change $>=1.5$ obtained from the integrative hypothesis testing method [33]. In order to determine whether a set of genes showed statistically significant and/or concordant differences between two biological states such as M1 versus M0-NM, GSEA software tool was applied to RNAseq dataset. Briefly, gene sets were obtained from MSigDB [34] or previously published analysis [16] Genes in the RNAseq dataset were sorted in descending order using the 'Signal2Noise' ranking metric and computed enrichment score (ES) using a Kolmogorov-Smirnov running sum statistic for the gene set. Significance was computed using a null distribution of the ES generated from a random gene set by 1000 permutations. For the training and evaluation of the classifier using the gene signatures, two principal dimensions using principal component analysis (PCA) were extracted from expression matrix of the gene signature and then SVM algorithm was applied to determine the discrimination border between the two groups (M1-poly versus M0-NM).

\section{Statistics}

The PNBX cohort $(n=99)$ was separated into five subcohorts based upon tumor burden at diagnosis (oligo or poly) or no tumor at diagnosis $+/$ - metastatic progression (oligo, poly, or no metastases) on at last follow-up (Supplementary Table 1). Bivariable analyses compared patient clinical characteristics across the sub-cohorts using Chi-square (or Fisher's exact) tests for categorical variables and ANOVA (or Wilcoxon rank-sum or median) tests for continuous variables. Kaplan-Meir survival analyses were performed for prostate cancer-specific and allcause mortality stratified by tumor burden categories described above. In addition, BCR-free survival was examined in high and low risk groups defined by the risk index using Cox proportional hazard regressions of the gene signature. Clinical characteristics were also compared between CIN status (high v. low) stratified by patient race (African-American and white). Lastly, multivariate logistic regression analyses were conducted for CRPC, prostate cancer-specific mortality and all-cause mortality to calculate odds ratios for CIN status (high v. low), controlling for age, race, PSA ( $>20 \mathrm{v} . \leq 20)$ and Gleason sum $(>8 \mathrm{v}$. $\leq 8$ ). Analyses were performed using Stata (StataCorp LP, College Station, TX, USA), SAS 9.4 (SAS Institute Inc., Cary, NC, USA), MATLAB (Mathworks, Natick, MA, USA) and R (v.3.1 http://www.r-project.org/).

\section{Results}

\section{CIN70 is significantly elevated in CRPC metastases}

The CIN70 signature was previously derived by identifying differentially expressed genes in tumors displaying high versus low levels of chromosomal imbalance. CIN70 was applied to five CRPC transcriptome datasets [22, 35-38]. The signature activation scores derived from primary tumors were compared to CRPC metastases (Fig. 1). CIN70 scores were significantly higher in CRPC metastases compared to primary tumors across all datasets.

\section{CIN70 score strongly correlates with various genomic alterations in the PC cohort in TCGA}

Since the high CIN70 signature activation score strongly associated with CRPC metastases, we sought to determine if it correlated with genomic evidence of CIN (i.e., broad CNAs) as well as unfavorable tumor features and outcome in untreated PC tissue samples. To assess the correlations of specific types of genomic aberrations and CIN70 activation scores measured in primary PC, we employed the PC cohort $(n=473)$ found in TCGA (Fig. 2a). We considered five different types of genomic 

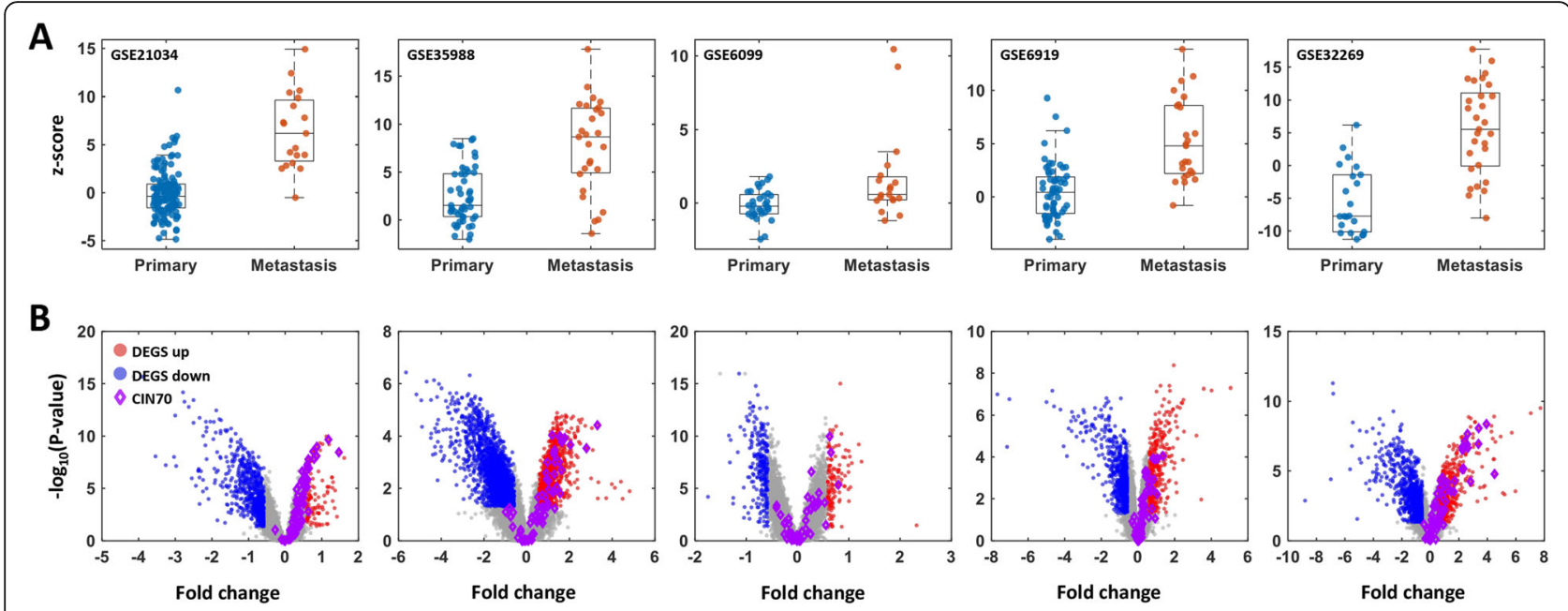

Fig. 1 CIN70 score is significantly higher in MCRPC compared to primary tumors. Five transcriptome data from independent PC cohorts that included primary tumors and MCRPC were analyzed for CIN70 activation score. a Box plots comparing primary tumors and metastatic tumors demonstrated overall differences in gene expression scores (rank-sum $p<0.00001$ for all). b Volcano plots reflecting differential expression in primary tumors versus metastases were annotated with CIN70 genes (purple) to highlight differential expression in CRPC metastases versus primaries

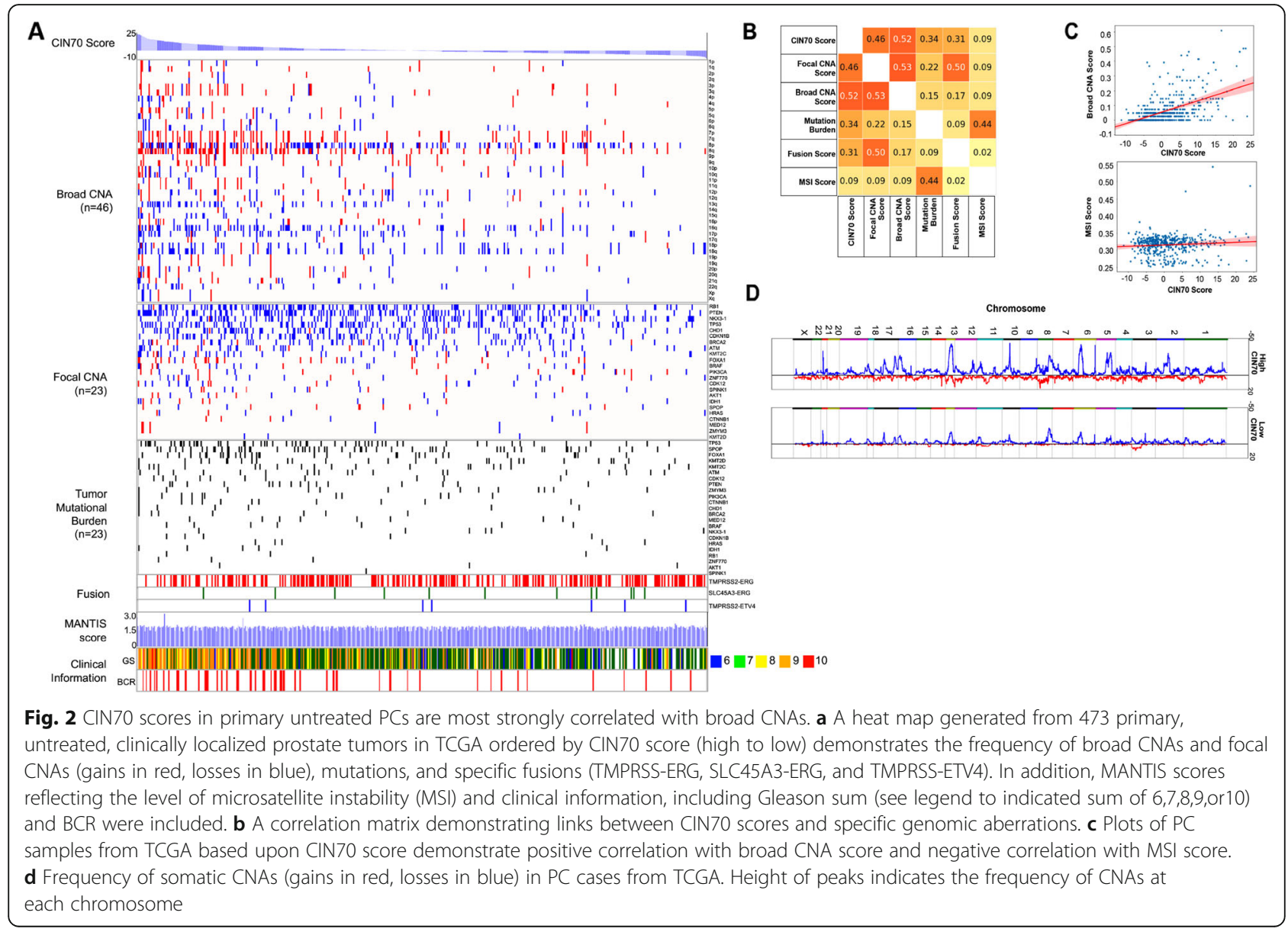


alterations including focal CNAs, broad CNAs, tumor mutational burden (TMB), gene fusions, and microsatellite instability (MSI) for each case. All genomic alterations were quantified using the individual scoring method (see Methods). Briefly, CNA events were identified in each sample and assigned broad or focal status based exclusively upon length using GISTIC 2.0 [26]. The TMB and fusion score were computed using the number of events identified in each case. The MSI score was based upon the MANTIS scoring method, and precomputed scores for the PC cohort in TCGA were obtained from a previously published report [27]. Cases were displayed in a heat map based upon CIN70 score (high to low) in order to visualize recurrent genomic alterations associated with the CIN (Fig. 2a). Pathology Gleason score and biochemical recurrence (BCR) status were also included on the map for each case. Comparing CIN scores to specific classes of genomic alterations allowed determination of correlation coefficients (Fig. 2b). The CIN70 score most strongly correlated with broad CNAs $(r=0.52$, Fig. 2c). There was a positive correlation with focal CNAs $(r=0.46)$, which was weaker with mutational burden $(r=0.34)$ and fusions $(r=0.31)$. No significant correlation between CIN70 score and MSI score was observed ( $r=0.09$, Fig. 2c). High CIN70 scores were associated with PC displaying Gleason score of 8 or higher (Additional File 1, Supplemental Fig. 1). PC cases displaying $\mathrm{BCR}$ were also more likely to display high CIN70 scores (Additional File 1, Supplemental Fig. 1). These findings confirm that high CIN70 score is reflective of broad CNA frequency in PC, which, in turn, is associated with aggressive disease and poor outcome in TCGA cases. To assess whether specific, recurrent CNAs differ between cases with high versus low CIN scores, TCGA cases classified as CIN70-high versus CIN70-low were compared (Fig. 2d). An increase in recurrent CNAs were identified throughout the genome, but no specific chromosomal locations where affected by increases in CNAs, confirming the global genomic impact of CIN.

\section{PNBX cohort}

Having established that CIN70 scores are highest in mCRPC and high-risk primary CSPCs contained in TCGA, we sought to evaluate transcriptomic profiles derived from untreated primary tumors of men diagnosed with de novo metastases (clinical stage M1). High-grade $\mathrm{PC}$ areas in PNBX were procured for RNA sequencing (RNAseq) from formalin-fixed and paraffin-embedded diagnostic PNBX of 99 patients (Fig. 3a). We accessed selected archival diagnostic PNBX from a racially and ethnically diverse cohort of 1927 men who were diagnosed and treated exclusively within a single Veterans Affairs (VA) healthcare system (Additional File 1, Supplemental Fig. 2). Sub-stratification of this cohort was performed based upon metastatic tumor burden (oligo versus poly) at diagnosis and follow-up (Additional File 1, Supplemental Fig. 2A). Kaplan-Meier curves demonstrated overall survival (Additional File 1, Supplemental Fig. 2B), with M1-poly and M0-poly cases displaying significantly shorter survival than M1-oligo and M0-oligo cases. For comparison, we selected high-grade PC cases without evidence of metastatic progression (M0-NM) over a median follow-up of 56 months. Clinical characteristics of the sequenced PNBX cases are provided in Additional File 1, Supplemental Table 1.

\section{mCRPC biology embedded in PNBX M1 cases}

We aimed to identify the transcriptomic footprint of metastatic disease in primary tumors by comparing mCRPC and primary tumors collected from PNBX of men diagnosed with de novo metastatic disease (M1oligo and M1-poly). We also questioned the amount of CIN in these cancers. Towards this goal, we identified 1234 DEGs by comparing RNA sequencing datasets of PCs from men in the VA cohort diagnosed with de novo metastatic disease (M1-poly or M1-oligo) versus nonmetastatic (M0-NM) cases. We also derived DEGs through comparison of gene expression between primary tumors from RPs and metastases collected at rapid autopsy in two published mCRPC cohorts (Taylor and Grasso data sets) $[22,35]$. Strong correlations were revealed between DEGs from the VA cohort and the DEGs derived from the Taylor $(r=0.64)$ or Grasso $(r=0.45)$ cohorts (Fig. 3b). Slightly weaker correlations were evident for M1-oligo cases ( $r=0.34$ and 0.27 , respectively).

Next, the overlap amongst DEGs across datasets (PNBX, Taylor, and Grasso) was used to identify 157 shared DEGs (Fig. 3c). Functional enrichment analysis in the 157 DEGs demonstrated the greatest activity of pathways associated with mitotic nuclear division, cell proliferation and cell-cell signaling (Fig. 3d). A large portion of DEGs $(89 / 157,57 \%)$ had the same directionality in gene expression between primary tumors associated with de novo metastases and CRPC sampled at metastatic sites. In a multidimensional scaling diagrams supervised by the 157 DEG set, cases without metastases (M0-NM) and M1 cases (both M1-oligo and M1-poly) form distinct clusters (Fig. 3e). Similarly, when applied to the Taylor and Grasso cohorts, primary tumors were separated from metastases (Fig. 3e). Collectively, these results demonstrate that untreated primary tumors from men with de novo metastases possess gene expression profiles that are correlative to heavily treated $\mathrm{mCRPC}$. These results suggest mCRPC biology is embedded in the primary tumors of M1 patients and has the potential to reveal biological mediators of metastases, castrationresistance and lethal $\mathrm{PC}$ at the time of diagnosis via standard-of-care PNBX. 


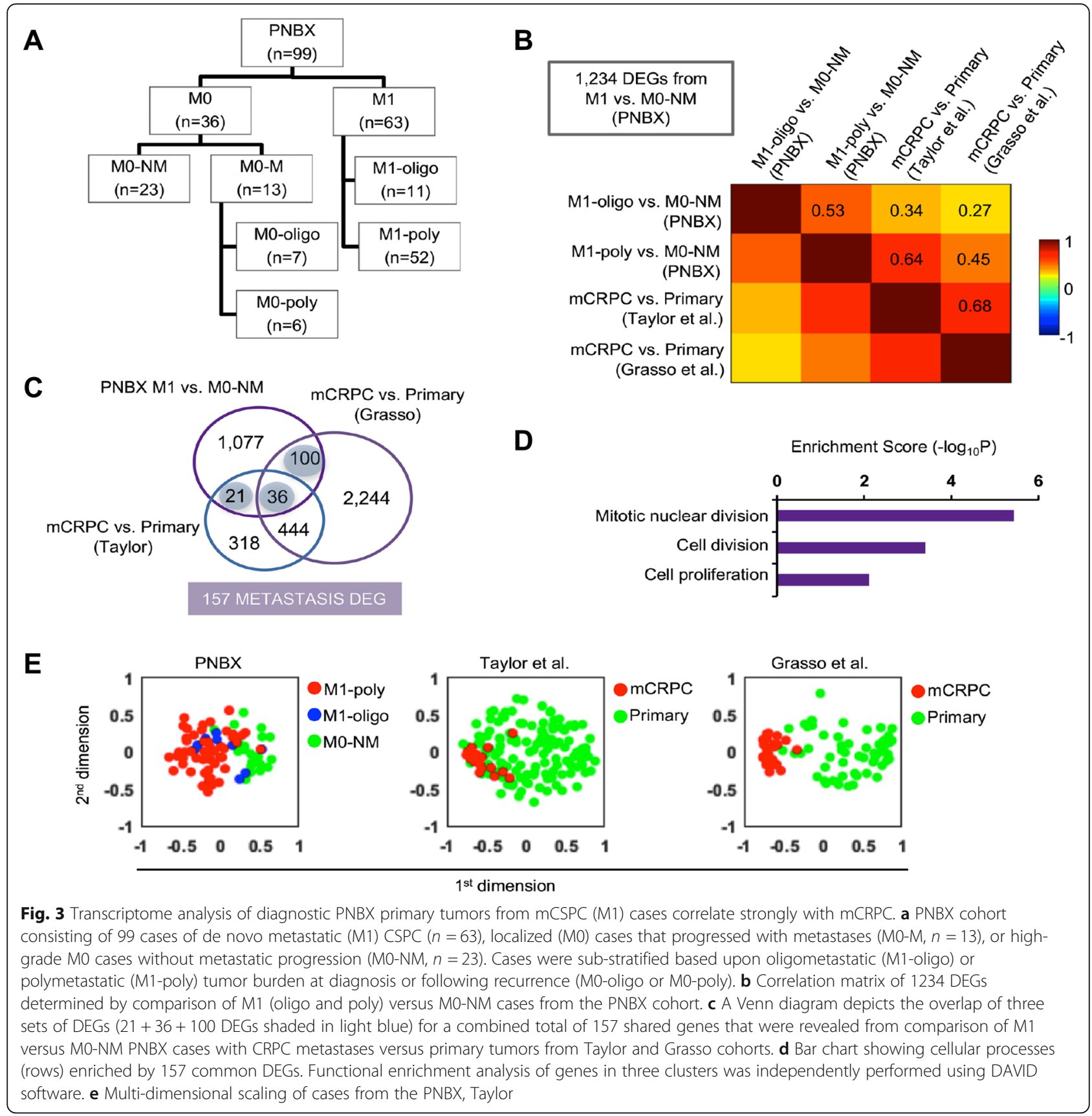

PNBX M1 cases represent high CIN without deregulated DDR We hypothesized that CIN70, which is highly elevated in mCRPC, may demonstrate a similar expression profile in M1 PNBX. Consequently, we evaluated enrichment of CIN70 gene expression via gene set enrichment analysis (GSEA) of M1 versus M0-NM cases. This analysis revealed significant up-regulation of CIN70 genes in M1 tumors (Additional File 1, Supplemental Fig. 3) [16]. In order for tumor cells to tolerate CIN, inactivation of the TP53 gene or its associated pathway is often required [39]. Interrogation of genes linked to the hallmark p53 pathway activation signature demonstrated downregulated in M1 relative to M0-NM tumors ( $p=$ 0.031). Interestingly, there was no enrichment in the DDR gene signature, indicating that this potential mechanism of genomic instability may not be prevalent in de novo metastatic CSPC (Additional File 1, Supplemental Fig. 3).

\section{Derivation of PC-CIN}

Since M1-oligo and M1-poly cases displayed significant differences in PC-specific survival (Additional File 1, 
Supplemental Fig. 1B), we hypothesized that distinct biological triggers may influence tumor burden. Consistent with this idea, out of total 1234 DEGs in M1 versus M0$\mathrm{NM}$, a relatively small portion of the DEGs (105 genes; 9\%) were common in both M1-oligo and M1-poly, while most of the genes (696/801 DEGs in M1-oligo and 433/ 538 DEG in M1-poly) were exclusively regulated in M1oligo or M1-poly cases (Additional File 1, Supplemental Fig. 4A). These DEGs were grouped into 3 clusters (shared, oligo-dominant, and poly-dominant) based on their differential expression patterns (Additional File 1, Supplemental Fig. 4B). To identify cellular processes within each group, functional enrichment analysis was performed using DAVID software (Additional File 1, Supplemental Fig. 4C) [40]. While the oligo-dominant cluster was enriched in inflammatory response, steroid metabolic processing, cell-cell signaling, and cell differentiation, the poly-dominant cluster displayed the strongest enrichment in cell proliferation and mitotic cell division. Consistent with this, GSEA analysis of M1poly versus M0-NM revealed a leading-edge subset of genes that significantly contributed to the enrichment of CIN70 and demonstrated significant up-regulation in M1 tumors (Fig. 4a and Additional File 1, Supplemental Fig. 3A). Notably, seven out of the top 19 leading-edge genes (PBK, CEP55, UBE2C, MELK, TPX2, PTTG1, and CDCA3) regulate mechanisms during mitosis [41]. We will refer to these seven genes as PC-CIN (prostate cancer-CIN). In order to determine whether the CIN70 signature genes or simplified PC-CIN associated with metastasis (M)-stage (M1 versus M0-NM) at diagnosis, we developed a prediction model using a support vector machine (SVM) algorithm (see Methods and Additional File 1, Supplemental Fig. 5) and tested its accuracy using PC-CIN or CIN70 genes. The model displayed a high level of accuracy in predicting

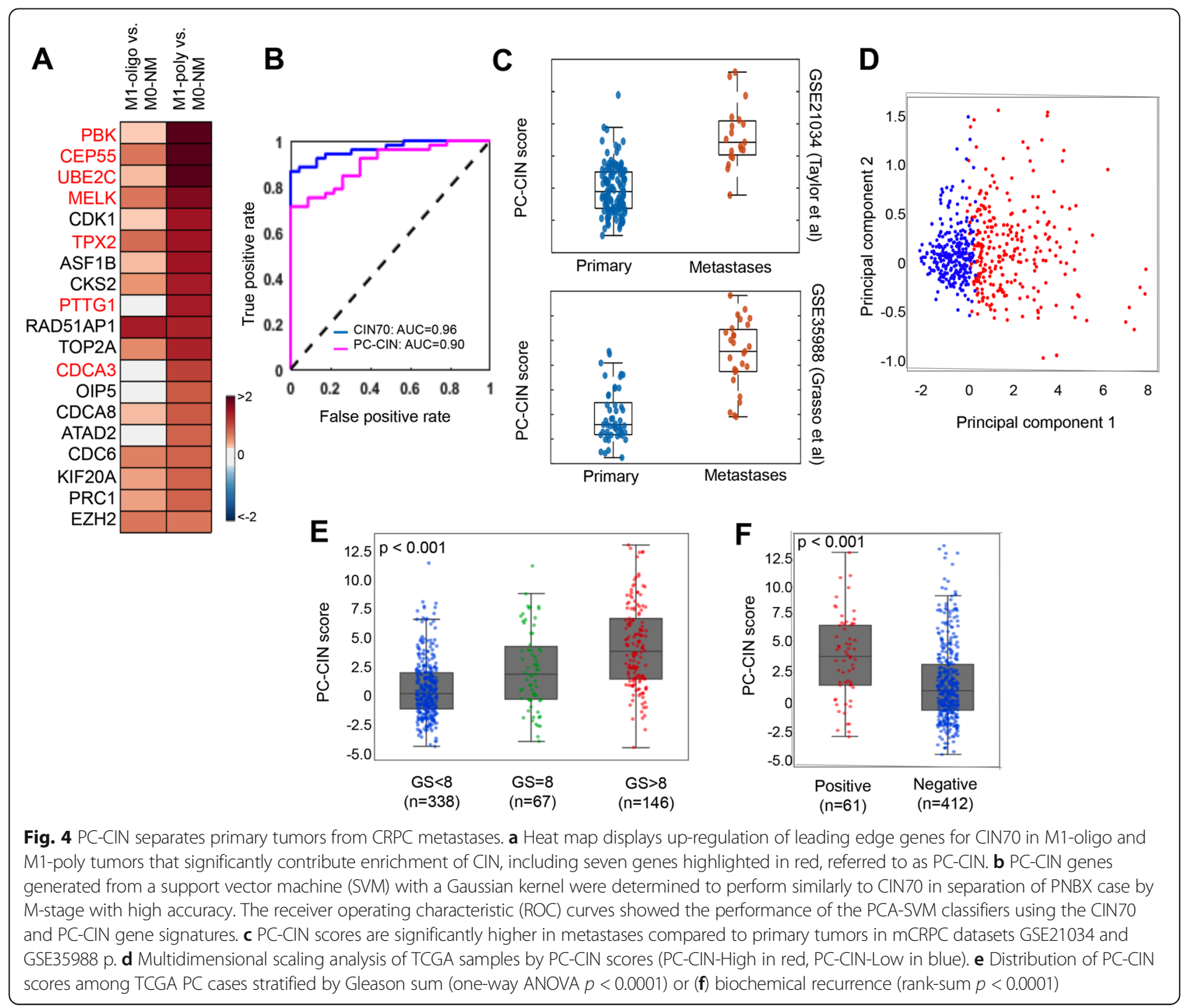


metastasis stage with area under the curve (AUC) value of 0.90 for PC-CIN and 0.96 for CIN70 (Fig. $4 \mathrm{~b})$. Both CIN70 and PC-CIN appeared significantly enriched in $\mathrm{mCRPC}$ relative to primary tumors across five datasets (Fig. 4c and Additional File 1, Supplemental Fig. 5A, all $p<0.00001$ ). PC-CIN activation score was also highest in Gleason 8 and higher PC samples in TCGA, as well as in cases of BCR (Fig. 4d-f).

PNBX analysis revealed heterogeneity of CIN in M1 cases To better understand the distribution of CIN70 and PCCIN scores in the context of the PNBX cohort $(n=99)$, we created an integrative heat map of CIN70 genes split into functional groups, as well as the 7 PC-CIN genes (Fig. 5a). Embedded in this heat map is the CIN70 score, with cases arranged from CIN70-low to CIN70-high, the disease stage (M0-NM, M0-oligo, M0-poly, M1-oligo, M1-poly), and the Gleason Sum (6-10). The heat map allows observation of the pattern of distribution of de novo metastatic (M1) cases along the spectrum of CIN scores. Interestingly, a bimodal distribution of M1 cases is observed, with 23/63 displaying CIN70 scores in the lowest third and 25/63 displaying CIN70 scores in the highest third. PC-CIN gene expression variability appeared to mirror the expression pattern of CIN70 genes. A volcano plot of PC-CIN genes in M0-NM versus M1poly cases in the PNBX cohort demonstrates differential expression (Fig. 5b). PC-CIN scores are significantly higher in M1-poly cases compared to M0-NM cases $(p=0.0426)$, however, the wide range of PC-CIN scores is evident in the box plot in Fig. 5c, which reflects the bimodal distribution of M1 CIN scores observed in the heat map (Fig. 5a).

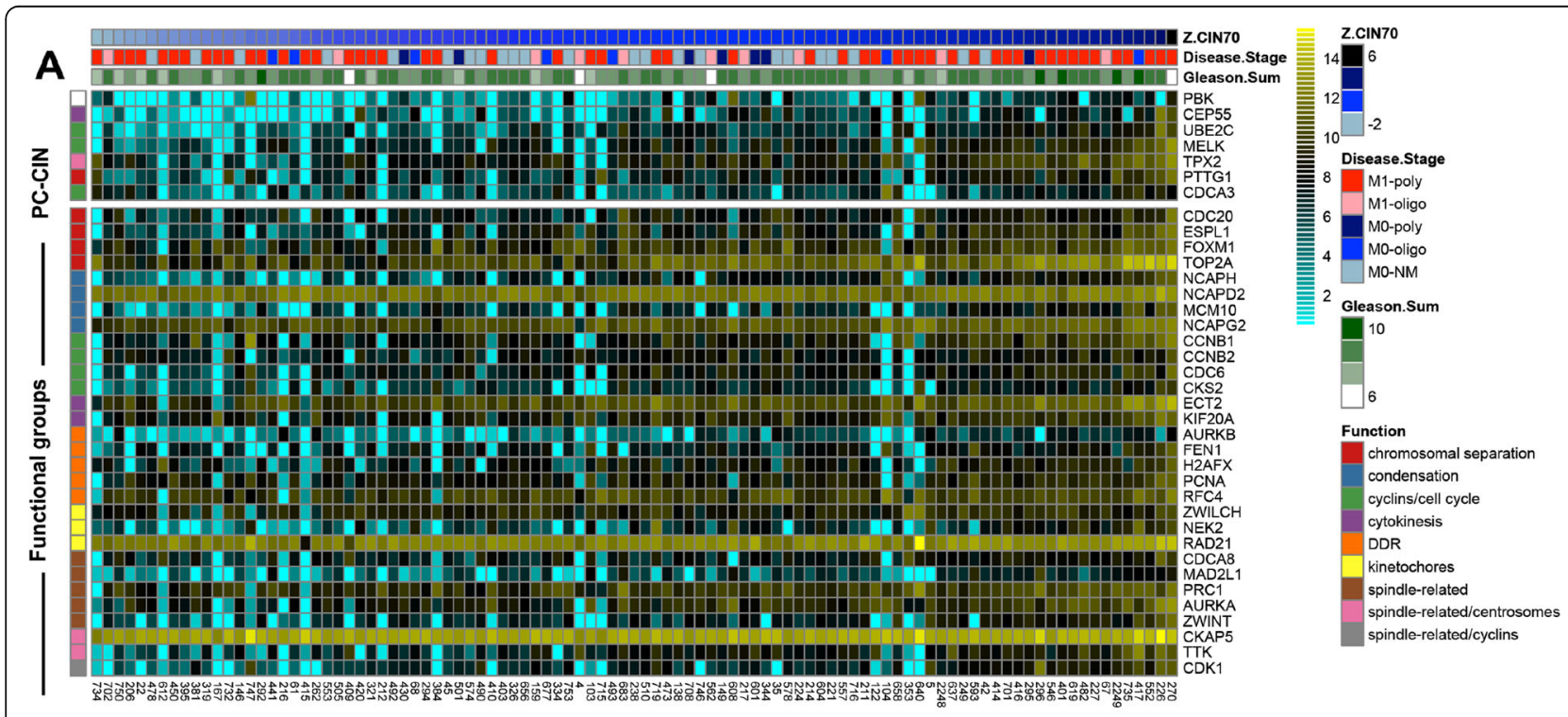

B

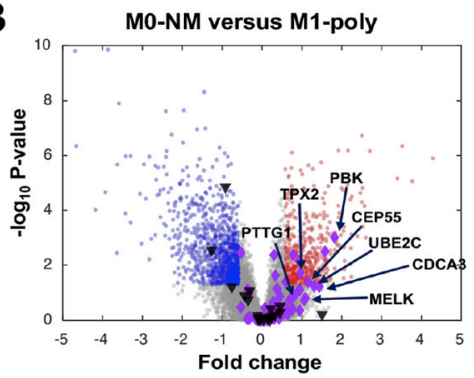

C

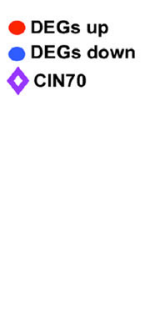

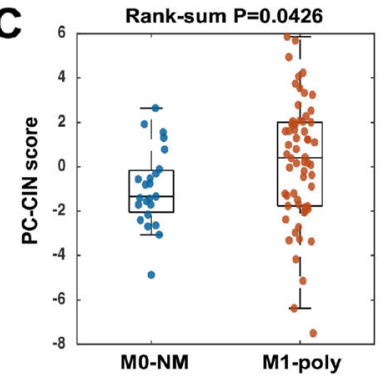

D

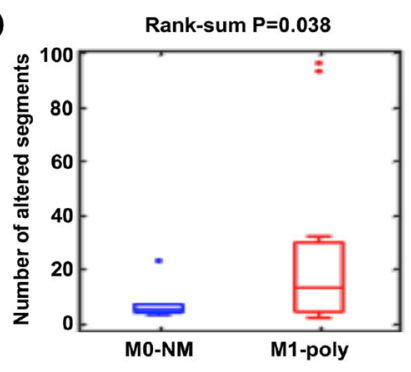

Fig. 5 CIN70 score highlights distinct M1 subtypes. Derivation of CIN70 score in individual samples in the PNBX cohort $(n=99)$ identifies bimodal distribution of M1 cases. a Heat map of PNBX samples organized by CIN70 score (low to high). Clinicopathological information is given in the header according to the legend (disease stage, Gleason sum). PC-CIN genes and specific genes from the CIN70 signature organized into functional groups are shown in accordance with the legend (chromosome replication, condensation, cyclins/cell cycle, cytokinesis, DDR, kinetochores, spindle-related, spindlerelated/centrosomes, and spindle-related/cyclins). $\mathbf{b}$ Volcano plot highlights distribution of PC-CIN genes among DEGS identified by comparison of M)NM and M1-poly cases from the PNBX cohort. c Boxplot demonstrates range of significantly higher PC-CIN scores found in M1-poly versus M1-NM PNBX cases. $\mathbf{d}$ Box plots demonstrate significantly higher frequency of CNAs found in select M1-poly versus MO-NM cases from the PNBX cohort 
To identify genomic evidence of CIN in M1 cases, we sampled the same tumor regions for DNA extraction that were previously selected to generate transcriptome data in M0-poly and M0-NM cases. Since there is limited tissue in PNBX, only 24 cases yielded sufficient quality/quantity of DNA for CNA evaluation. However, a significant increase $(p=0.038)$ in copy number alterations in M1-poly versus M0-NM cases was observed in this small sample (Fig. 5d), consistent with heightened frequencies of amplifications and deletions associated with CIN in TCGA (Fig. 2). Gain of MYC and loss of RB1 and SIAH3 were also identified, consistent with previous studies of genetic alterations associated with poor PC prognosis (Additional File 1, Supplemental Fig. 6A and B) [42-44].

\section{Differentially expressed genes in CIN-high versus CIN-low cases}

The bimodal distribution of M1 cases when organized by CIN70 score suggests both CIN-dependent and CINindependent gene associations and processes linked to PC lethality. Consequently, we evaluated DEGs and biological processes associated with CIN70-Low versus CIN70-High, as well as PC-CIN-Low versus PC-CINHigh cases from the PNBX cohort. Heat maps of the DEGs based upon these different gene expression signatures is displayed in Additional File 1, Supplemental Fig. 7A and B). Enriched biological processes associated with CIN70 and PC-CIN scores (low versus high) are also displayed (Additional File 1, Supplemental Fig. 7C and D). Distinct biological processes appear to be active in CIN-high versus CIN-low tumors. As expected, CINhigh tumors involve processes associated with cell cycle, mitosis, and chromosome segregation. In contrast, the top processes associated with CIN-low tumors involve developmental signatures, specifically those related to vascular and urogenital system development, as well as muscle contraction. In a subgroup analysis of clinical, pathological, and outcomes variables associated with $\mathrm{CIN}$ - high versus CIN-low metastatic tumors, there were no significant differences found in de novo metastatic cases. When all metastatic cases were included (i.e., those that were initially diagnosed as M0, but then progressed with metastasis), CIN-high cases were more likely to have pathological N1 stage and demonstrated significantly poorer outcomes than CIN-low cases (Supplemental Tables 4 \&5).

Additional analysis of previously described CIN genes and drivers was also performed. During chromosome segregation, sister chromatids are separated by a kinetochore mediated attachment to spindle microtubules [45]. The microtubules are nucleated from centromeres, which require the highly evolutionary conserved OIP5/ MIS18 $\beta$ for proper assembly [46]. Disruption of kinetochore and centrosome dynamics are components of neoplastic transformation, and, similar to aneuploidy, centrosome amplification is another hallmark of cancer [47]. Although there was no clear evidence of specific functional group dysregulation among nine mechanistic subgroups of CIN70 genes that we annotated (chromosomal separation, condensation, cyclins/cell cycle, DNA damage repair (DDR), kinetochores, spindle-related, spindle related/centrosomes, and spindle related/cyclins), we did find interesting expression differences that connect CIN and metastatic progression. KIF20A is one of the leading edge genes found on GSEA of M1-poly versus MO-NM DEGs (Fig. 4a) and is homologous to KIF2B, a protein that directly promotes tumor metastasis in cell line models of CIN [15]. Both KIF20A and KIF2B are significantly overexpressed in PC-CIN-high cases relative to (Additional File 1, Supplemental Fig. 8A).

A recent analysis of highly aneuploidy breast cancers in TCGA found overexpression of three transcriptional regulators, E2F1, MYBL2, and FOXM1 [13]. Overexpression of these genes in non-transformed Xenopus embryos was sufficient to significantly increase the rate of chromosomal missegregation and initiate aneuploidy. Evaluation of expression of these transcription factors in CIN70-high versus CIN70-low PNBX demonstrated significantly elevated expression in CIN70-high cases (Additional File 1, Supplemental Fig. 8B). In addition, 6/7 PC-CIN genes (CEP55, UBE2C, MELK, TPX2, PTTG1, and CDCA3) were also found to be among the top DEGs identified through comparison of high aneuploidy versus low aneuploidy breast tumors in TCGA. These results suggest that the same drivers and effectors are involved across tumor types.

\section{Staging and prognostic value of PC-CIN in independent cohorts}

Next, we questioned whether the PC-CIN signature genes were associated with disease progression in presumed localized (M0) cases. PC-CIN score separated high- and low-risk BCR groups from two independent PC cohorts (Fig. 6a) [22, 48]. We also tested the ability of PC-CIN to separate cases based upon M-stage equally well in subcohorts of African-American (AA) and European-American (EA) PNBX $(n=121)$. Clinical characteristics of patients included in this expanded PNBX cohort are shown in Additional File 1, Supplemental Table 2. PC-CIN was associated with metastatic progression in cases stratified by race with AUC of 0.78 for AA men and 0.80 for EA men (Fig. 6b). Both AA and EA men displayed significantly higher PC-CIN in M1 PNBX compared to M0 PNBX $(P=1.06 \mathrm{e}-04$ and $3.11 \mathrm{e}-04$, respectively, Fig. $6 c)$. In both racial groups, univariate analyses demonstrated that PC-CIN high cases were significantly more likely to be classified as clinical stage M1, 


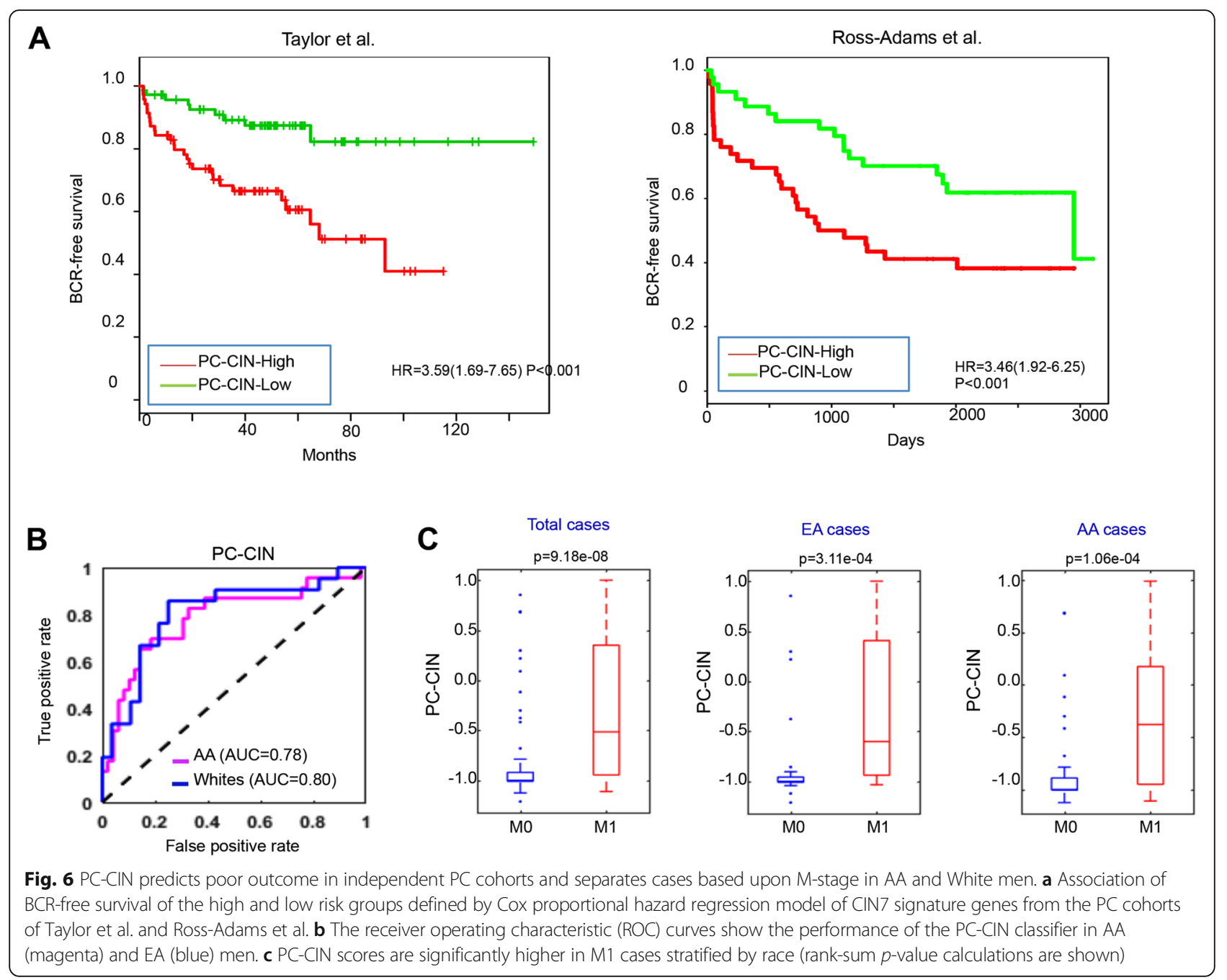

progress to CRPC, and die from PC (Additional File 1, Supplemental Table 3). In order to evaluate the impact of PC-CIN score in the context of other clinicopathological variables, multivariate logistic regression analysis was performed. After controlling for age, race, PSA and Gleason sum, PC-CIN-high is significantly associated with higher odds of M1 stage, CRPC, PC-death and all-cause mortality in multivariate analysis (OR 10.84, 16.13, 6.26 and 6.00 , respectively; all $p<0.001$, Table 1 ).

\section{Discussion}

We used a bioinformatics approach to rank PC tumors in TCGA based upon CIN70 signature activation scores and found a positive correlation between CIN70 score and frequency of aneuploidy events (whole chromosome and large fragments of chromosome gains/losses). We evaluated genomic and transcriptomic evidence of CIN across datasets representing the full clinical spectrum of $\mathrm{PC}$, including localized CSPC, $\mathrm{mCSPC}$, and $\mathrm{mCRPC}$,

Table 1 Multivariate logistic regression analysis of variables associated with PC progression

\begin{tabular}{|c|c|c|c|}
\hline & CRPC & PC-Death & All-Cause Mortality \\
\hline PC-CIN High Versus Low & $16.13(3.23,80.55)$ & $6.26(2.44,16.04)$ & $6.00(2.45,14.71)$ \\
\hline Age at Diagnosis & $0.93(0.86,1.01)$ & $1.00(0.95,1.05)$ & $1.01(0.97,1.06)$ \\
\hline Race (AA v. White) & $0.99(0.22,4.96)$ & $0.31(0.11,0.86)$ & $0.43(0.16,1.11)$ \\
\hline PSA $\left(<20 v_{.}>20\right)$ & $1.04(0.22,4.96)$ & $8.23(3.06,22.15)$ & $5.71(2.29,14.25)$ \\
\hline Gleason (> 8 v. $<8)$ & $2.29(0.52,10.03)$ & $0.62(0.23,1.64)$ & $0.87(0.35,2.18)$ \\
\hline
\end{tabular}


and found increased CIN70 scores in mCRPC. Comparison of diagnostic biopsies (PNBX) from patients with de novo metastatic (M1) CSPC and published MCRPC datasets revealed a common gene set with CIN70 genes, that could be simplified into a 7-gene classifier (PC-CIN) capable of predicting metastatic stage, poor outcomes (BCR, CRPC progression, metastasis), and lethality (PC-specific and all cause mortality) in PC cohorts.

Genomic instability in cancer results from ongoing DNA damage and is a fundamental feature of many aggressive tumors, including mCRPC [49]. Recent profiling studies and clinical trials in PC have focused on identifying mutations and structural rearrangements mediated by defective DNA damage repair, because these aberrations may render tumors susceptible to PARP inhibitors and immunotherapy $[5,6,50-52]$. However, CINinduced broad copy number alterations and DNA damage from ruptured micronuclei byproducts also contribute to overall genomic instability in cancer and have the potential to drive tumor evolution, metastases and the emergence of treatment-resistant populations [53]. Support of this concept in PC was provided in a recent study examining aneuploidy in archival specimens from surgical cohorts with localized disease that had longterm follow-up outcome variables available [23]. In this analysis, the authors identified aneuploid PC tumors in TCGA and generated a transcriptomic profile that correlated with lethal progression in the independent cohorts. Using a different computational approach, our findings affirm that arm-level gains and losses, measured indirectly through the transcriptome via, are linked to PC progression. We also employed Oncoscan in a subset of cases in order to directly measure copy number alterations. Since we were confined to using the diagnostic PNBX tissues, which contain small amounts of cancer, we could not always perform orthogonal studies to confirm aneuploidy. This is a limitation of this study. However, by performing RNA sequencing, we were able to implicate functional categories of genes directly involved in chromosome separation that are dysregulated in tumors exhibiting CIN, as well as potential transcriptomic drivers of this process. Altogether, our study has illuminated a tumor landscape where genomic aberrations can rapidly accumulate and drive cancer progression.

Despite a renewed focus on mCSPC and movement of treatments previously reserved for mCRPC to the frontline of mCSPC care, the 5-year survival of patients presenting de novo with metastatic PC is still low [24]. Combination therapies are primarily focused on targeting the androgen axis and incapable of eliciting cures, indicating the need for novel approaches [54-58]. CIN70 and the distilled PC-CIN signatures reveal, in both metastatic CSPC and $\mathrm{mCRPC}$, that deregulated expression of genes involved in chromosomal segregation during anaphase is linked to metastatic progression and lethality. Interestingly, factors that may be capable of initiating the vicious cycle of CIN through centrosome disruption have been studied previously for links with PC progression and centrosome genes/mediators are present on CIN70/ PC-CIN gene lists [59-61]. Bisphenol-A (BPA) is an alkaphenyl estrogen-like compound found in plastics, including food containers and baby bottles. Low dose exposure to BPA appears to be associated with in increased incidence of $\mathrm{PC}$ and known to induce irreversible changes in centrosomes [61-63].

Targeting CIN directly is complicated. One way to target CIN is to restore the activity of mitotic checkpoints that stall cell proliferation when they sense CIN. Another approach is to identify drugs with synthetic lethality to CIN. Inhibitors of kinases that regulate the duplication of centrosomes, by itself can induce CIN. Drugs that inhibit targets broadly associated with CIN, such as polo-like kinases, cyclindependent kinases, and Aurora kinases are being explored in clinical trials, and histone deacetylases (HDAC1, HDAC5, and SIRT1) are being evaluated for efficacy in inhibiting centrosome duplication and amplification [61, 64-66]. Interestingly, taxols, in addition to inhibiting depolymerization of microtubules and blocking mitosis, are reported to affect the capacity of centrosomes to nucleate microtubules, which may explain another mechanism of anti-tumor activity [67]. Recent clinical trials in PC revealed significant improvement in overall survival in M1-poly patients receiving docetaxel combined with standardof-care ADT [54, 55]. As we know from our analysis, a significant subset of M1-poly CSPC cases, similar to the ones enrolled in the clinical trials, demonstrates CIN $[55,68]$. M1-poly cases in our cohort were predominantly distributed at the extremes of the CIN70/ PC-CIN score spectrum (the majority were classified as either PC-CIN-High or PC-CIN-Low), so we would predict that the CIN-high subset of M1 cases is likely to be more responsive to taxol than the CIN-low subset. Consequently, hormone-sensitive metastatic cases that are CIN-high may be optimally managed by including docetaxel in the combination therapy regimen with ADT. This prediction can be explored in future studies, however, it will be necessary to understand the mechanisms of metastatic progression and lethality exhibited by CIN-low tumors, as well. It is possible that CIN-low tumors display plasticity through epigenetic mechanisms that support survival and progression.

There is an unmet need of measuring the magnitude of CIN-mediated aneuploidy in PC using cost-effective, 
reliable, and feasible assays [23]. Our discovery of a 7gene PC-specific PC-CIN classifier constitutes a small gene panel is quantifiable through RNA sequencing of cancer tissue retrieved from archival diagnostic PNBX. Consequently, the PC-CIN classifier may lead to more cost-effective measurements of aneuploidy. For example, the application of new technologies, such as Nanostring ${ }^{\circ}$, could facilitate the measurement of the PC-CIN7 gene signature and be used to develop a clinical grade assay. It is also possible that CIN gene expression could be measured in the urine. We are currently in the process of determining whether or not CIN can be measured in diagnostic H\&E slides using machine-learning image analysis technology. These opportunities for measurement and validation of CIN in PC have the potential to offer improved, staging, prognostic, and possibly predictive information for management of newly diagnosed PC patients, without the need to access additional tissue samples.

\section{Conclusions}

Quantitating CIN in PNBX by transcriptome profiling is feasible and has the potential to serve as a biomarker that identifies patients with a high risk of lethal progression at the time of diagnosis. Additionally, CIN indicates a specific biological feature of a subset of metastatic prostate cancers that can be further defined and explored for therapeutic targeting in the future.

\section{Supplementary information}

Supplementary information accompanies this paper at https://doi.org/10. 1186/s12885-020-06817-1.

Additional file 1.

\footnotetext{
Abbreviations

AA: African-American; ANOVA: Analysis of Variance; AUC: Area Under the Curve; BCR: Biochemical Recurrence; CIN: Chromosomal instability; CIN70: 70gene CIN signature; CGas-STING: cyclic GMP-AMP synthase-stimulator of interferon genes; CNAs: Copy number alterations; CT: Computed Tomography Scan; CRPC: Castration-Resistant Prostate Cancer; CSPC: Castration-Sensitive Prostate Cancer; DEGs: Differentially expressed genes; DDR: DNA Damage Repair; EA: European American; ES: Enrichment Score; FFPE: Formalin Fixed Paraffin Embedded; ${ }^{18} \mathrm{~F}-\mathrm{NaF}$ PET: ${ }^{18} \mathrm{~F}-\mathrm{NaF}$ positron emission tomography; GLA-VA: Greater Los Angeles Veterans Affairs Healthcare System; GSEA: Gene Set Enrichment Score; H\&E: Hematoxylin and Eosin; IRB: Institutional Review Board; PC: Prostate Cancer; M0: Clinical metastasis stage (no metastases present); MO-M: stage M0 at diagnosis then progressed with metastases; MO$\mathrm{NM}$ : stage $\mathrm{M0}$ at diagnosis with no metastatic progression; M1: Clinical metastasis stage (metastases present); MANTIS: Microsatellite Analysis for Normal-Tumor Instability; mCSPC: Metastatic Castration-Sensitive Prostate Cancer; mCRPC: Metastatic Castration-Resistant Prostate Cancer; MRI: Magnetic Resonance Imaging; MSI: Microsatellite instability; MX: equivocal M stage at diagnosis; Oligo: Oligometastatic prostate cancer; PCA: Principal Component Analysis; PC-CIN: 7-gene CIN signature; PNBX: Prostate needle biopsies; Poly: Polymetastatic prostate cancer; PSA: Prostate Specific Antigen; SNP: Single Nucleotide Polymorphism;

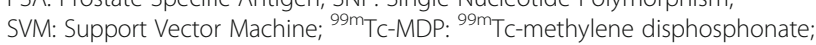
TCGA: The Cancer Genome Atlas; TMB: Tumor mutational Burden
}

\section{Acknowledgements}

We would like to acknowledge and thank the VA Greater Los Angeles Healthcare System and the Veterans that we serve for supporting this work.

\section{Authors' contributions}

E.M. identified cases, acquired and analyzed data, and contributed to the study design, manuscript writing and review. S.Y. supervised all computational analysis, contributed to the study design, manuscript preparation and review. R.C. provided clinical and technical support and acquired data. M. K \& J.Y. performed data analysis and prepared figures for the manuscript. S.L. provided clinical support for the study and contributed to manuscript review. X.L. acquired genomic and transcriptomic data, assisted in data analysis. L.K. performed statistical analysis and managed the clinical database. J.H. provided support for sequencing. M.J. performed data analysis and created Figs. C.G. assisted with study design, direction of the project, data analysis, and manuscript review. M.L. supervised tissue procurement, slide annotation, and block transfer, performed data analysis, and manuscript review. B.K., assisted with slide annotation, interpretation of results, manuscript preparation, and direction of the study. M.F. contributed to manuscript preparation, direction of the study, and data interpretation. I.G. founded and directed the archival biopsy analysis project, supervised all data collection and quality control, analyzed data, acquired funding, and wrote the manuscript. EM and SY contributed equally to the study and are designated co-first author. The author order is alphabetical. All authors have read and approved the manuscript.

\section{Funding}

The funding sources include: Jean Perkins Foundation, Prostate Cancer Foundation (17CHAL04 and Creativity Award), Movember Foundation (GAP6 Unique TMAs), Stephen Spielberg Foundation, Department of Defense (PC131996 and PC130244), and National Institute of Health (P01 CA09891209, U54 CA143931, R01 CA131255, P50 CA092131). None of the aforementioned funding agencies played a role in the design of the study, collection, analysis, or interpretation of data or in the writing of the manuscript.

\section{Availability of data and materials}

Gene expression data and OncoScan CNA data were deposited into the GEO database with accession GSE147493 and GSE147353, respectively.

\section{Ethics approval and consent to participate}

We received institutional review board (IRB) approval to abstract data and procure both fresh and archival PNBX samples from men diagnosed and treated within the Greater Los Angeles VA (GLA-VA) Healthcare System (protocol numbers PCC2018-020201 and PCC2010-11489). Informed consent was obtained for all prospectively collected tissue specimens. A consent waiver was approved for collection of archival samples and data. All specimens were stripped of personal health information and identifiers.

\section{Consent for publication}

Not Applicable.

\section{Competing interests}

There are not competing interests to disclose.

\section{Author details}

${ }^{1}$ Department of Urology, David Geffen School of Medicine at UCLA, Box 951738, 10833 Le Conte Ave 66-188 CHS UCLA, Los Angeles, CA 90095, USA. ${ }^{2}$ Department of Biomedical Sciences, Cedars-Sinai Medical Center, California, Los Angeles, USA. ${ }^{3}$ Department of Surgery, Cedars-Sinai Medical Center, California, Los Angeles, USA. ${ }^{4}$ Department of Medicine, Division of Hematology-Oncology, David Geffen School of Medicine at UCLA, California, Los Angeles, USA. ${ }^{5}$ Department of Pathology, David Geffen School of Medicine at UCLA, California, Los Angeles, USA. ${ }^{6} J o n s s o n$ Comprehensive Cancer Center, David Geffen School of Medicine at UCLA, Box 951738, 10833 Le Conte Ave 66-188 CHS UCLA, Los Angeles, CA 90095, USA. ${ }^{7}$ Department of Pathology, Greater Los Angeles Veterans Affairs Health System, California, Los Angeles, USA. ${ }^{8}$ Division of Urology, Greater Los Angeles Veterans Affairs Healthcare Center, Box 951738, 10833 Le Conte Ave 66-188 CHS UCLA, Los Angeles, CA 90095, USA. 
Received: 16 January 2020 Accepted: 2 April 2020

Published online: 07 May 2020

\section{References}

1. Janssen A, et al. Chromosome segregation errors as a cause of DNA damage and structural chromosome aberrations. Science. 2011;333(6051):1895-8.

2. McGranahan N, et al. Cancer chromosomal instability: therapeutic and diagnostic challenges. EMBO Rep. 2012;13(6):528-38.

3. Negrini S, Gorgoulis VG, Halazonetis TD. Genomic instability--an evolving hallmark of cancer. Nat Rev Mol Cell Biol. 2010;11(3):220-8.

4. Hanahan D, Weinberg RA. Hallmarks of cancer: the next generation. Cell. 2011;144(5):646-74.

5. Lengauer C, Kinzler KW, Vogelstein B. Genetic instabilities in human cancers. Nature. 1998;396(6712):643-9.

6. Luijten MNH, Lee JXT, Crasta KC. Mutational game changer: Chromothripsis and its emerging relevance to cancer. Mutat Res. 2018;777:29-51.

7. Giam M, Rancati G. Aneuploidy and chromosomal instability in cancer: a jackpot to chaos. Cell Div. 2015;10:3.

8. Shen MM. Chromoplexy: a new category of complex rearrangements in the cancer genome. Cancer Cell. 2013;23(5):567-9.

9. Hatch EM, et al. Catastrophic nuclear envelope collapse in cancer cell micronuclei. Cell. 2013;154(1):47-60.

10. Godek KM, Compton DA. Quantitative methods to measure aneuploidy and chromosomal instability. Methods Cell Biol. 2018;144:15-32.

11. Taylor AM, et al. Genomic and functional approaches to understanding Cancer aneuploidy. Cancer Cell. 2018;33(4):676-89 e3.

12. Oltmann J, et al. Aneuploidy, TP53 mutation, and amplification of MYC correlate with increased intratumor heterogeneity and poor prognosis of breast cancer patients. Genes Chromosomes Cancer. 2018;57(4):165-75.

13. Pfister $K$, et al. Identification of drivers of aneuploidy in breast tumors. Cell Rep. 2018;23(9):2758-69.

14. Davoli, T., et al., Tumor aneuploidy correlates with markers of immune evasion and with reduced response to immunotherapy. Science. 2017;355(6322):1-14.

15. Bakhoum SF, et al. Chromosomal instability drives metastasis through a cytosolic DNA response. Nature. 2018;553(7689):467-72.

16. Carter $\mathrm{SL}$, et al. A signature of chromosomal instability inferred from gene expression profiles predicts clinical outcome in multiple human cancers. Nat Genet. 2006;38(9):1043-8.

17. Lee HS, et al. A new assay for measuring chromosome instability (CIN) and identification of drugs that elevate CIN in cancer cells. BMC Cancer. 2013;13:252

18. Pritchard CC, et al. Inherited DNA-repair gene mutations in men with metastatic prostate Cancer. N Engl J Med. 2016;375(5):443-53.

19. Mateo J, et al. DNA repair in prostate Cancer: biology and clinical implications. Eur Urol. 2017;71(3):417-25.

20. Mateo J, et al. DNA-repair defects and Olaparib in metastatic prostate Cancer. N Engl J Med. 2015;373(18):1697-708.

21. Goodall J, et al. Circulating cell-free DNA to guide prostate Cancer treatment with PARP inhibition. Cancer Discov. 2017;7(9):1006-17.

22. Taylor BS, et al. Integrative genomic profiling of human prostate cancer. Cancer Cell. 2010;18(1):11-22

23. Stopsack KH, et al. Aneuploidy drives lethal progression in prostate cancer. Proc Natl Acad Sci U S A. 2019;116(23):11390-5.

24. Siegel RL, Miller KD, Jemal A. Cancer statistics, 2018. CA Cancer J Clin. 2018; 68(1):7-30.

25. D'Amico AV, et al. Cancer-specific mortality after surgery or radiation for patients with clinically localized prostate cancer managed during the prostate-specific antigen era. J Clin Oncol. 2003;21(11):2163-72.

26. Mermel $\mathrm{CH}$, et al. GISTIC2.0 facilitates sensitive and confident localization of the targets of focal somatic copy-number alteration in human cancers. Genome Biol. 2011;12(4):R41.

27. Kautto EA, et al. Performance evaluation for rapid detection of pan-cancer microsatellite instability with MANTIS. Oncotarget. 2017;8(5):7452-63.

28. Gao Q, et al. Driver fusions and their implications in the development and treatment of human cancers. Cell Rep. 2018;23(1):227-38 e3.

29. Levine DM, et al. Pathway and gene-set activation measurement from mRNA expression data: the tissue distribution of human pathways. Genome Biol. 2006;7(10):R93.

30. Liao Y, Smyth GK, Shi W. The subread aligner: fast, accurate and scalable read mapping by seed-and-vote. Nucleic Acids Res. 2013;41(10):e108.
31. Liao Y, Smyth GK, Shi W. featureCounts: an efficient general purpose program for assigning sequence reads to genomic features. Bioinformatics. 2014;30(7):923-30.

32. Robinson MD, Oshlack A. A scaling normalization method for differential expression analysis of RNA-seq data. Genome Biol. 2010;11(3):R25.

33. Hwang $D$, et al. A data integration methodology for systems biology. Proc Natl Acad Sci U S A. 2005;102(48):17296-301.

34. Liberzon A, et al. Molecular signatures database (MSigDB) 3.0. Bioinformatics. 2011;27(12):1739-40.

35. Grasso CS, et al. The mutational landscape of lethal castration-resistant prostate cancer. Nature. 2012;487(7406):239-43.

36. Tomlins SA, et al. Integrative molecular concept modeling of prostate cancer progression. Nat Genet. 2007;39(1):41-51.

37. Yu YP, et al. Gene expression alterations in prostate cancer predicting tumor aggression and preceding development of malignancy. J Clin Oncol. 2004; 22(14):2790-9.

38. Cai $C$, et al. ERG induces androgen receptor-mediated regulation of SOX9 in prostate cancer. J Clin Invest. 2013;123(3):1109-22.

39. Gronroos E, Lopez-Garcia C. Tolerance of chromosomal instability in Cancer: mechanisms and therapeutic opportunities. Cancer Res. 2018; 78(23):6529-35.

40. Huang da W, Sherman BT, Lempicki RA. Systematic and integrative analysis of large gene lists using DAVID bioinformatics resources. Nat Protoc. 2009; 4(1):44-57.

41. Giotti, B., et al., Assembly of a parts list of the human mitotic cell cycle machinery. J Mol Cell Biol. 2018.

42. Robbins CM, et al. Copy number and targeted mutational analysis reveals novel somatic events in metastatic prostate tumors. Genome Res. 2011;21(1):47-55.

43. Thangavel C, et al. RB loss promotes prostate Cancer metastasis. Cancer Res. 2017;77(4):982-95.

44. Fleming $\mathrm{WH}$, et al. Expression of the c-myc protooncogene in human prostatic carcinoma and benign prostatic hyperplasia. Cancer Res. 1986;46(3):1535-8.

45. Cheerambathur DK, et al. Crosstalk between microtubule attachment complexes ensures accurate chromosome segregation. Science. 2013;342(6163):1239-42.

46. Wang J, et al. Mitotic regulator Mis18beta interacts with and specifies the centromeric assembly of molecular chaperone Holliday junction recognition protein (HJURP). J Biol Chem. 2014;289(12):8326-36.

47. Fiszman ML, Luchelli-Fortis MA, Stefano FJ. Amphetamine antagonizes the presynaptic inhibitory effect of clonidine through an interaction at the level of the alpha 2-adrenoceptors. Gen Pharmacol. 1989;20(3):351-8.

48. Ross-Adams $\mathrm{H}$, et al. Integration of copy number and transcriptomics provides risk stratification in prostate cancer: a discovery and validation cohort study. EBioMedicine. 2015;2(9):1133-44.

49. Tapia-Laliena MA, et al. High-risk prostate cancer: a disease of genomic instability. Urol Oncol. 2014;32(8):1101-7.

50. Boysen $\mathrm{G}$, et al. SPOP mutation leads to genomic instability in prostate cancer. Elife. 2015;4.

51. Brown JS, et al. Targeting DNA repair in Cancer: beyond PARP inhibitors. Cancer Discov. 2017;7(1):20-37.

52. Mouw KW, et al. DNA damage and repair biomarkers of immunotherapy response. Cancer Discov. 2017;7(7):675-93.

53. Michor F, Iwasa Y, Nowak MA. Dynamics of cancer progression. Nat Rev Cancer. 2004;4(3):197-205.

54. James ND, et al. Addition of docetaxel, zoledronic acid, or both to first-line long-term hormone therapy in prostate cancer (STAMPEDE): survival results from an adaptive, multiarm, multistage, platform randomised controlled trial. Lancet. 2016;387(10024):1163-77.

55. Sweeney CJ, et al. Chemohormonal therapy in metastatic hormone-sensitive prostate Cancer. N Engl J Med. 2015;373(8):737-46.

56. Davis ID, et al. Enzalutamide with standard first-line therapy in metastatic prostate Cancer. N Engl J Med. 2019;381(2):121-31.

57. Chi KN, et al. Apalutamide for metastatic, castration-sensitive prostate Cancer. N Engl J Med. 2019;381(1):13-24.

58. Fizazi $\mathrm{K}$, et al. Abiraterone acetate plus prednisone in patients with newly diagnosed high-risk metastatic castration-sensitive prostate cancer (LATITUDE): final overall survival analysis of a randomised, double-blind, phase 3 trial. Lancet Oncol. 2019;20(5):686-700.

59. Pihan GA, et al. Centrosome defects can account for cellular and genetic changes that characterize prostate cancer progression. Cancer Res. 2001;61(5):2212-9.

60. Ho SM, et al. Bisphenol a and its analogues disrupt centrosome cycle and microtubule dynamics in prostate cancer. Endocr Relat Cancer. 2017;24(2):83-96. 
61. Schatten H, Ripple MO. The impact of centrosome pathologies on prostate Cancer development and progression. Adv Exp Med Biol. 2018;1095:67-81.

62. Tarapore $\mathrm{P}$, et al. Exposure to bisphenol a correlates with early-onset prostate cancer and promotes centrosome amplification and anchorageindependent growth in vitro. PLoS One. 2014;9(3):e90332.

63. Can A, Semiz O, Cinar O. Bisphenol-a induces cell cycle delay and alters centrosome and spindle microtubular organization in oocytes during meiosis. Mol Hum Reprod. 2005;11(6):389-96.

64. Cheung $\mathrm{CH}$, et al. Aurora kinase inhibitor patents and agents in clinical testing: an update (2011 - 2013). Expert Opin Ther Pat. 2014;24(9):1021-38.

65. Schoffski P. Polo-like kinase (PLK) inhibitors in preclinical and early clinical development in oncology. Oncologist. 2009;14(6):559-70.

66. Ling $\mathrm{H}$, et al. Suppression of centrosome duplication and amplification by deacetylases. Cell Cycle. 2012;11(20):3779-91.

67. De Brabander $\mathrm{M}$, et al. Taxol induces the assembly of free microtubules in living cells and blocks the organizing capacity of the centrosomes and kinetochores. Proc Natl Acad Sci U S A. 1981;78(9):5608-12.

68. van Soest RJ, de Wit R. Irrefutable evidence for the use of docetaxel in newly diagnosed metastatic prostate cancer: results from the STAMPEDE and CHAARTED trials. BMC Med. 2015:13:304.

\section{Publisher's Note}

Springer Nature remains neutral with regard to jurisdictional claims in published maps and institutional affiliations.

Ready to submit your research? Choose BMC and benefit from:

- fast, convenient online submission

- thorough peer review by experienced researchers in your field

- rapid publication on acceptance

- support for research data, including large and complex data types

- gold Open Access which fosters wider collaboration and increased citations

- maximum visibility for your research: over $100 \mathrm{M}$ website views per year

At BMC, research is always in progress.

Learn more biomedcentral.com/submissions 